\title{
Acute Oral Toxicity and Kinetic Behaviors of Inorganic Layered Nanoparticles
}

\author{
Jin Yu, Hea-Eun Chung, and Soo-Jin Choi \\ Department of Food Science and Technology, Seoul Women's University, Seoul 139-774, Republic of Korea \\ Correspondence should be addressed to Soo-Jin Choi; sjchoi@swu.ac.kr
}

Received 25 June 2013; Revised 5 September 2013; Accepted 5 September 2013

Academic Editor: Xiaoming Li

Copyright (C) 2013 Jin Yu et al. This is an open access article distributed under the Creative Commons Attribution License, which permits unrestricted use, distribution, and reproduction in any medium, provided the original work is properly cited.

\begin{abstract}
Layered double hydroxide (LDH) nanoparticles, also known as anionic clays, have attracted a great deal of interest for their potential as delivery carriers. Recent studies showed that LDH nanoparticles can efficiently deliver drugs or bioactive molecules into cells, which are highly related to their endocytic pathway. However, the efficient cell permeation capacity of LDH may also raise concern about their toxicity potential. In this study, the acute oral toxicity of LDH nanoparticles was assessed, and their kinetic behaviors, such as plasma concentration-time curve, tissue distribution, and excretion, were also evaluated in mice. No significant effects of oral LDH nanoparticles on behaviors, body weight gain, survival rate, and organosomatic index were observed up to the dose of $2000 \mathrm{mg} / \mathrm{kg}$ for 14 days. Serum biochemical parameters did not significantly increase, indicating that LDH nanoparticles did not cause acute liver or kidney injury. Plasma concentration of LDH nanoparticles rapidly decreased within 30 min depending on exposure doses, but they did not accumulate in any specific organ. Their excretion via urine and feces was observed within $24 \mathrm{~h}$. These findings suggest that LDH nanoparticles do not exhibit acute oral toxicity and favorable kinetic behaviors in mice and, therefore, will be promising candidates for biological and pharmaceutical applications.
\end{abstract}

\section{Introduction}

In recent years, inorganic nanomaterials have been extensively developed for biomedical applications, such as diagnostic or imaging agents, tissue engineering, and drug delivery carriers [1-3]. Nanoparticles have large surface areas and high reactivity resulting from their small size. Moreover, their structure can be easily modified for target specific delivery [4]. This is fascinating to efficiently deliver target molecules to specific organs and consequently contributing to enhanced efficacy as well as reduced toxicity. Among them, layered double hydroxides (LDHs) nanoparticles, also known as anionic clays or hydrotalcite-like compounds, consist of positively charged metal hydroxide sheets and interlayers containing charge-balancing anions and water molecules to form lamellar structure $[5,6]$. Thus, LDHs can intercalate anionic molecules, such as drugs, nucleotides, and biomolecules, into the interlayer spaces, release them in a controlled manner, and eventually protect them against harsh biological conditions, thereby attracting much attention as nonviral delivery carriers $[7,8]$. Recent studies have demonstrated that LDHs can efficiently deliver anticancer drugs or bioactive molecules into cells, leading to enhanced efficacy of intercalated molecules, which are closely related to their energydependent endocytic pathway, clathrin-mediated endocytosis [9-11]. However, the efficient cell permeation capacity of LDHs may cause undesirable harmful effects by interfering with biological cellular functions, which raises concern about their toxicity potential on human health.

Toxicological effects of LDH nanoparticles on human cells were recently investigated in vitro, demonstrating low cytotoxicity of LDHs compared to other inorganic nanoparticles such as iron oxide, silica, and carbon nanotubes [12]. Cytotoxicity of LDHs with respect to particle size [13], chemical composition [14], and structural stability [15] was also reported; ZnAl-LDH and $50 \mathrm{~nm}$ particles showed slightly higher toxicity than MgAl-LDH and 100 to $350 \mathrm{~nm}$ particles, respectively, and carbonate form of $\mathrm{LDH}$ with strong stability exhibited high toxicity compared to its chloride form possessing easy decomposition property in cell lines [16]. However, 


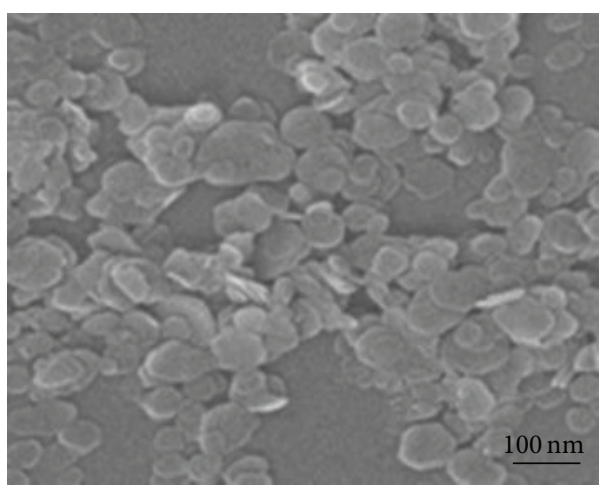

(a)

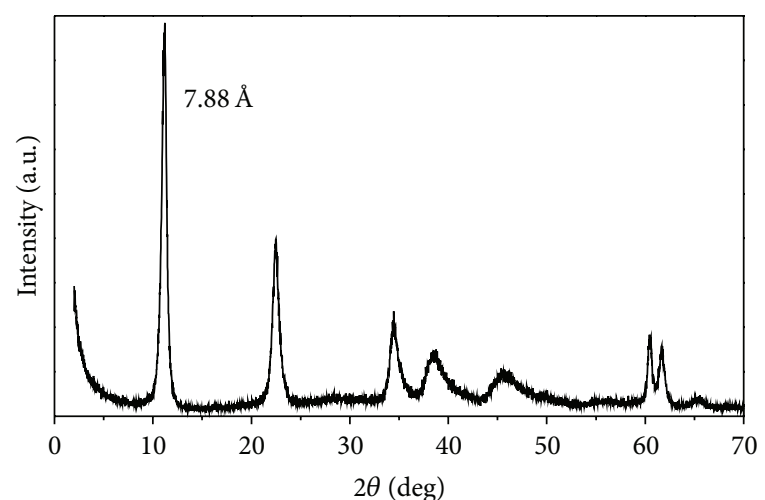

(b)

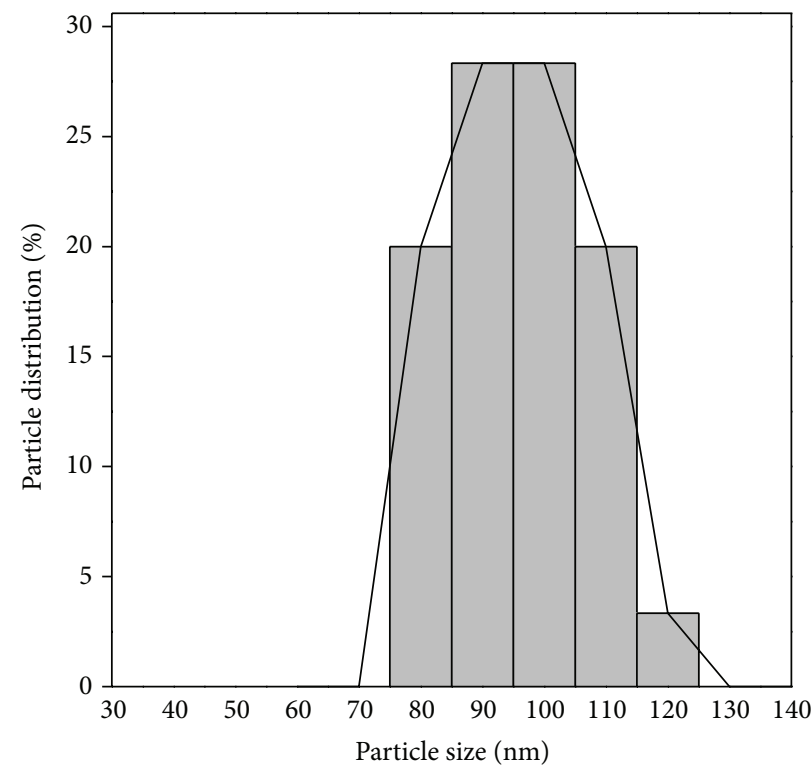

(c)

FIGURE 1: SEM image (a), PXRD pattern (b), and size distribution (c) of LDH nanoparticles. Particle size distribution was presented by randomly selecting 200 particles from SEM image and measuring each particle size.

research on toxicity evaluation of LDHs in vivo is extremely limited. Toxicity evaluation in whole animals provides more practical data, reflecting interaction of nanoparticles with immune systems, proteins, and dynamic body fluids at the systemic level. In particular, no information about acute oral toxicity of LDHs is available, although the gastrointestinal tract is one of the important routes by which nanoparticles get into the human body, for example, via oral intake of water and foods or drugs delivered by nanomaterials $[17,18]$. Moreover, pharmacokinetics of LDHs have not been determined yet, which elucidate absorbed or accumulating amount as well as clearance kinetic profile in vivo.

In this study, the acute oral toxicity of LDH nanoparticles was evaluated in mice according to the OECD guideline. Pharmacokinetic, tissue distribution, and excretion studies were also performed after single-dose oral administration, aiming at understanding the relationships between toxicity potential of LDH nanoparticles and their kinetic behaviors in the body.

\section{Materials and Methods}

2.1. Preparation and Characterization of Nanoparticles. $\mathrm{LDH}$ nanoparticle, a chloride form of MgAl-LDH, was prepared by coprecipitation method; the salts $\mathrm{MgCl}_{2} \cdot 6 \mathrm{H}_{2} \mathrm{O}$ and $\mathrm{AlCl}_{3} \cdot 6 \mathrm{H}_{2} \mathrm{O}$, in a molar ratio $\mathrm{Mg} / \mathrm{Al}=2$, were dissolved in decarbonated water and then precipitated by addition of $\mathrm{NaOH} / \mathrm{NaCl}$ at a final $\mathrm{pH}$ of 10.5 . The suspension was hydrothermally treated for $12 \mathrm{~h}$ at $100^{\circ} \mathrm{C}$. The obtained white precipitation was centrifuged, washed with deionized water, and then freeze dried. Thus, prepared LDH nanoparticles were analyzed by powder X-ray diffraction (PXRD: Phillips PW3710 diffractometer with Ni-filtered $\mathrm{CuK} \alpha$ radiation $(\lambda=$ $1.5418 \AA$ )) and by scanning electron microscopy (SEM: HITACHI S-4300).

2.2. Animals and Diets. Male Balb/c mice, aged 7 weeks and weighing 25-27 g, were purchased from G-Bio (Seoul, Republic of Korea). The animals were housed in plastic 
laboratory animal cages in a ventilated room. The room was maintained at $20 \pm 2^{\circ} \mathrm{C}$ and $60 \pm 10 \%$ relative humidity on a $12 \mathrm{~h}$ light/dark cycle. Water and commercial laboratory complete food for mice were available ad libitum. They were acclimated to this environment for 7 days before receiving experimental treatment. All animal experiments were performed in compliance with the animal and ethics review committee of the Seoul Women's University.

2.3. Acute Toxicity Evaluation. The experiments to determine the $\mathrm{LD}_{50}$ (lethal dose $50 \%$ ) values of $\mathrm{LDH}$ nanoparticles were designed in accordance with the method provided by the OECD guideline 423. Prior to dosing, food but not water was withheld for $4 \mathrm{~h}$. LDH nanoparticles were homogeneously suspended in $0.9 \%$ saline by vortexing and ultrasonicating the suspension for $5 \mathrm{~min}$ at $42 \mathrm{kHz}$ (Vibra Cell Sonics \& Materials Inc.). Four different concentrations of LDH nanoparticles $(5,50,300$, and $2000 \mathrm{mg} / \mathrm{kg})$ were administered to each group of three mice via oral gavage in a stepwise procedure. Group of three mice, receiving identical volume of $0.9 \%$ saline, was served as a control group. Changes in body weight, behaviors, and symptoms were carefully recorded every day following the treatment. After 14 days, all organs were collected and organosomatic index was calculated by the following formula: [weight ( $\mathrm{g}$ ) of the organ/total body weight $(\mathrm{g})] \times 100$.

2.4. Blood Biochemical Analysis. Blood samples were also collected via tail vein, and the serum was obtained by centrifugation of the whole blood at $3000 \mathrm{rpm}$ for $10 \mathrm{~min}$. Liver function was evaluated based on the serum levels of alkaline phosphatase (ALP: ABD Bioquest), alanine aminotransferase (ALT: Bioo Scientific), and aspartate aminotransferase (AST: Bioo Scientific) according to the manufacturer's protocols. Nephrotoxicity was determined by measuring the concentrations of blood urea nitrogen (BUN: BioAssay Systems) and uric acid (UA: BioAssay Systems) in the serum according to the manufacturer's protocols.

2.5. Pharmacokinetics, Tissue Distribution, and Excretion Studies. For pharmacokinetic study, the blood samples (about $0.2 \mathrm{~mL}$ ) were collected via tail vein at several time points $(0,5,15,30,60,120$, and $240 \mathrm{~min})$ after single-dose oral administration of LDH nanoparticles $(5,50,300$, and $2000 \mathrm{mg} / \mathrm{kg}$ ). The blood sample at $0 \mathrm{~h}$ before oral administration was used to determine basal $\mathrm{Mg}$ level in the plasma. The blood samples were centrifuged at $3000 \mathrm{rpm}$ for $15 \mathrm{~min}$ at $4^{\circ} \mathrm{C}$ to obtain the plasma and stored at $-70^{\circ} \mathrm{C}$ before analysis. The following pharmacokinetic parameters were estimated using Kinetica program (version 4.4, Thermo Electron Corporation, USA): maximum concentration $\left(C_{\max }\right)$, time to maximum concentration $\left(T_{\max }\right)$, area under the plasma concentration-time curve (AUC), half-life $\left(T_{1 / 2}\right)$, and mean residence time (MRT). To evaluate biodistribution of LDH nanoparticles, the tissue samples such as brain, heart, kidney, liver, lung, and spleen were collected at $0,5,15,30$, and 60 min following euthanasia by $\mathrm{CO}_{2}$. Urine and feces were also collected for 7 days.

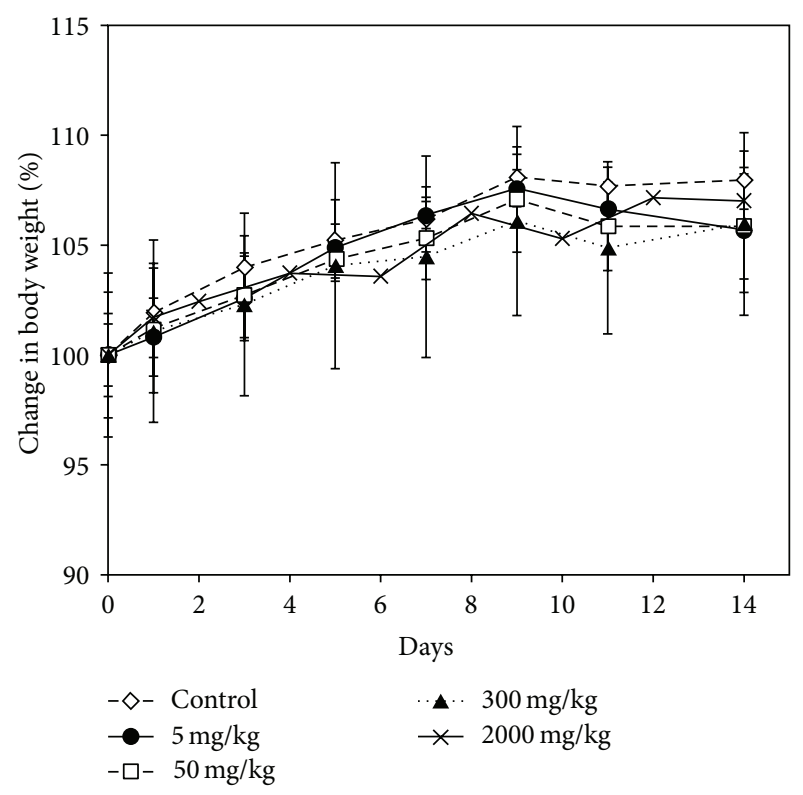

FIgure 2: Changes in body weight in mice administered four different doses of LDH nanoparticles for 14 days.

2.6. ICP-AES Analysis. The plasma, tissue samples, urine, and feces were digested in $3 \mathrm{~mL}$ of ultrapure nitric acid. After adding $0.5 \mathrm{~mL}$ of $\mathrm{H}_{2} \mathrm{O}_{2}$, each mixture was heated at about $160^{\circ} \mathrm{C}$ until the samples were completely digested. Then, the remaining nitric acid was removed by heating until the solutions were colorless and clear. The solutions were finally diluted to $10 \mathrm{~mL}$ with ultrapure water. Inductively coupled plasma-atomic emission spectroscopy (ICP-AES, Jobin Yvon Hriba, JY 2000 Ultrace) was used to analyze Mg concentration, a main component of $\mathrm{LDH}$, in the samples.

2.7. Statistical Analysis. Statistical analysis was performed using Student's $t$ test for unpaired data, and $P$ values of less than 0.05 were considered significant. All data were presented as mean \pm standard deviation (SD).

\section{Results and Discussions}

3.1. Characterization. The prepared LDH nanoparticles were analyzed by SEM (Figure 1(a)), showing homogenous size distribution with an average particle size of $91.8 \pm 10 \mathrm{~nm}$ (Figure 1(c)). PXRD pattern showed typical characteristic peaks for LDH nanoparticles (Figure 1(b)), with the 003 reflection around $\sim 11^{\circ}(2 \theta)$ and $d$-spacing of $7.88 \AA$. From the SEM image and PXRD pattern, average aspect ratio was determined to be 3.5 as calculated by particle diameter/particle thickness, which is well consistent with the previous report [10]. LDH nanoparticles of about $100 \mathrm{~nm}$ in size were synthesized because it was found to be optimum for efficient cellular uptake [10].

3.2. Acute Toxicity. Figure 2 shows change in body weight after single-dose oral administration of LDH nanoparticles in mice. No significant difference in body weight gain was 


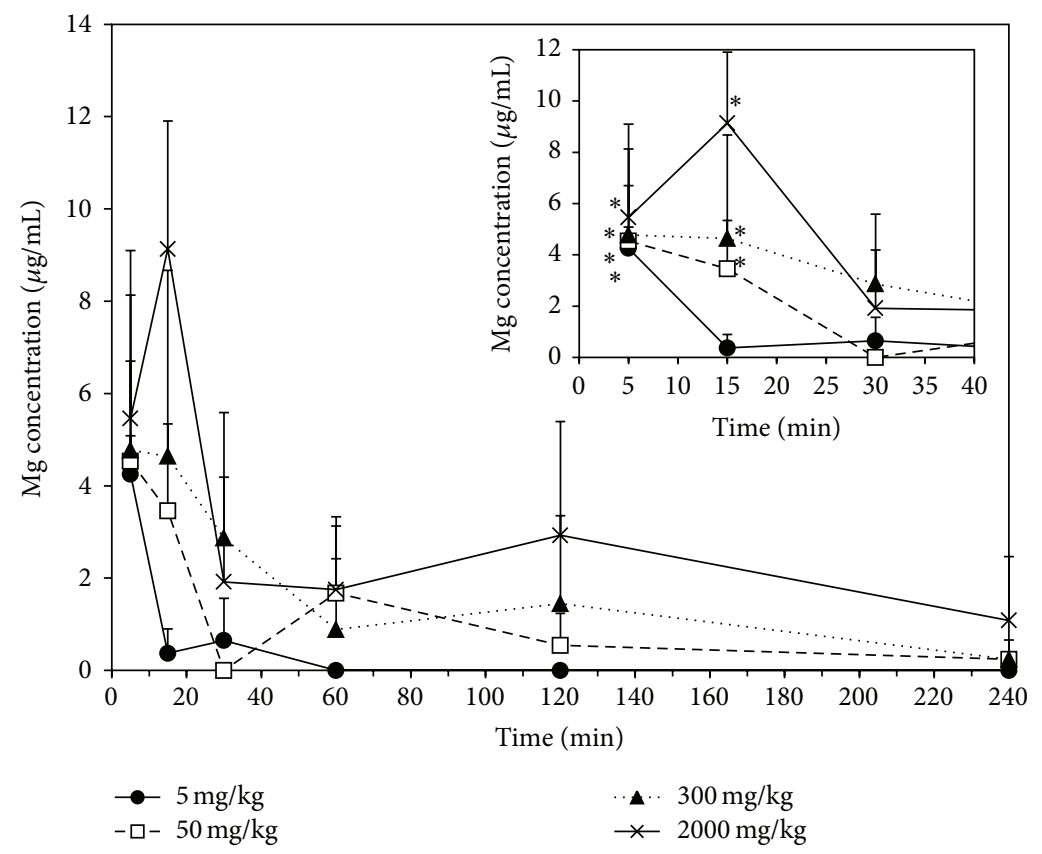

FIGURE 3: Plasma concentration-time curve of LDH nanoparticles after single-dose oral administration to mice. LDH concentrations were measured by analyzing a main component of $\mathrm{LDH}, \mathrm{Mg}$, using ICP-AES. The data were presented as increased Mg levels after substraction of basal $\mathrm{Mg}$ level in untreated control group. Insert: higher magnification of plasma concentration-time curves for 30 min. ${ }^{*}$ Significant difference from the control group $(P<0.05)$.

found between the control and the groups administered different doses of LDH nanoparticles $(P>0.05)$. Moreover, no mortality and abnormal behaviors or symptoms, such as decrease in food and water intake, diarrhea, loss of movement, and change in the size of eye's pupils and eye pigment, were observed in mice treated with even the highest dose for 14 days, indicating that the $\mathrm{LD}_{50}$ values were more than $2000 \mathrm{mg} / \mathrm{kg}$. This result is an indication of low oral toxicity potential of LDH nanoparticles. Organosomatic indices of organs including brain, heart, intestine, kidney, liver, lung, spleen, and stomach were also assessed following oral administration of LDH nanoparticles (Table 1), showing no significant decrease or increase of organosomatic indices of all organs in mice administered up to $2000 \mathrm{mg} / \mathrm{kg}$. This indicates that LDH nanoparticles did not affect any organ weight. All the results including changes in body weight, survival rate, and organosomatic index clearly suggest that orally administered LDH nanoparticles did not cause acute toxicity in mice.

3.3. Change in Blood Biochemical Parameters. Biochemical assay is generally and clinically used to evaluate targetspecific tissue injury or disease. Elevated levels of ALP, ALT, or AST in the blood are an indication of the liver damage and have been associated with liver injury, since these metabolic enzymes are primarily expressed in the liver $[18,19]$. On the other hand, urea, a major end product of protein catabolism in animals, is primarily produced in the liver and secreted by the kidney. A waste product generated from the degradation of purines, UA, is also filtered and removed by the kidney.
Thus, BUN or UA determination in the blood is very useful to assess kidney function $[18,20]$. Table 2 showed that the levels of all biochemical parameters in the serum of mice treated with high doses, 300 and $2000 \mathrm{mg} / \mathrm{kg}$ of $\mathrm{LDH}$ nanoparticles, did not significantly increase in comparison with those in the control group after 14 days $(P>0.05)$. Biochemical parameters were analyzed after 14 days to evaluate possible delayed toxicity after reabsorption in the intestine as well as to examine the capacity of recovery of these organs after exposure to $\mathrm{LDH}$ nanoparticles. No effect of $\mathrm{LDH}$ nanoparticles on biochemical parameters was also found after $24 \mathrm{~h}$ (data not shown). This result clearly suggests that LDH nanoparticles did not cause acute liver or kidney injury by gastrointestinal ingestion.

3.4. Pharmacokinetics. Pharmacokinetic behaviors of oral $\mathrm{LDH}$ nanoparticles in mice are presented in Figure 3. The plasma concentration-time curve, measured by a main component of $\mathrm{LDH}, \mathrm{Mg}$, was dependent on the dose administered. However, significant increase in LDH levels was found only within $15 \mathrm{~min}$ in all the cases, and the peak concentrations rapidly decreased after $30 \mathrm{~min}$ even when $2000 \mathrm{mg} / \mathrm{kg}$ were administered. The pharmacokinetic parameters following oral administration were presented in Table 3. It is clear that all parameters increased as administered dose increased. Based on AUC values which elucidate the total amount of nanoparticles reaching the systemic circulation, absorptions (\%) of 5, 50, 300, and $2000 \mathrm{mg} / \mathrm{kg}$ of $\mathrm{LDH}$ nanoparticles were determined to be about $2.85,1.37,0.40$, and $0.14 \%$, respectively. This result indicates that small amount of $\mathrm{LDH}$ 
TABLE 1: Organosomatic indices of organs in different groups of mice at 14 days after oral administration.

\begin{tabular}{|c|c|c|c|c|c|}
\hline Organ & Control & $5 \mathrm{mg} / \mathrm{kg}$ & $50 \mathrm{mg} / \mathrm{kg}$ & $300 \mathrm{mg} / \mathrm{kg}$ & $2000 \mathrm{mg} / \mathrm{kg}$ \\
\hline Brain & $0.88 \pm 0.15$ & $0.91 \pm 0.11$ & $0.79 \pm 0.15$ & $0.88 \pm 0.09$ & $0.86 \pm 0.07$ \\
\hline Heart & $0.44 \pm 0.12$ & $0.34 \pm 0.06$ & $0.35 \pm 0.02$ & $0.24 \pm 0.10$ & $0.29 \pm 0.04$ \\
\hline Intestine & $7.04 \pm 0.49$ & $6.03 \pm 0.33$ & $6.34 \pm 0.36$ & $5.59 \pm 1.00$ & $5.89 \pm 0.90$ \\
\hline Kidney & $0.93 \pm 0.11$ & $0.88 \pm 0.01$ & $0.81 \pm 0.02$ & $0.78 \pm 0.03$ & $0.82 \pm 0.03$ \\
\hline Liver & $3.81 \pm 0.37$ & $3.66 \pm 0.28$ & $3.33 \pm 0.19$ & $3.51 \pm 0.13$ & $3.35 \pm 0.09$ \\
\hline Lung & $0.37 \pm 0.02$ & $0.41 \pm 0.05$ & $0.41 \pm 0.05$ & $0.41 \pm 0.10$ & $0.37 \pm 0.02$ \\
\hline Spleen & $0.24 \pm 0.09$ & $0.23 \pm 0.04$ & $0.27 \pm 0.03$ & $0.21 \pm 0.05$ & $0.28 \pm 0.02$ \\
\hline Stomach & $0.51 \pm 0.17$ & $0.23 \pm 0.07$ & $0.65 \pm 0.24$ & $0.58 \pm 0.14$ & $0.42 \pm 0.26$ \\
\hline
\end{tabular}

All treated groups showed statistically no significant differences from the control group $(P>0.05)$.

TABLE 2: Changes in biochemical parameters in the serum of mice administered LDH nanoparticles.

\begin{tabular}{lccccc}
\hline Group & ALP $(\mathrm{U} / \mathrm{L})$ & ALT $(\mathrm{U} / \mathrm{L})$ & AST $(\mathrm{U} / \mathrm{L})$ & BUN $(\mathrm{mmol} / \mathrm{L})$ & $\mathrm{UA}(\mu \mathrm{mol} / \mathrm{L})$ \\
\hline Control & $120.30 \pm 28.05$ & $72.54 \pm 22.65$ & $166.52 \pm 43.76$ & $3.46 \pm 1.01$ & $156.81 \pm 38.21$ \\
$300 \mathrm{mg} / \mathrm{kg}$ & $91.51 \pm 33.77$ & $80.13 \pm 25.39$ & $152.22 \pm 39.92$ & $3.62 \pm 1.08$ & $145.55 \pm 29.52$ \\
$2000 \mathrm{mg} / \mathrm{kg}$ & $112.54 \pm 17.51$ & $85.76 \pm 29.61$ & $159.37 \pm 39.97$ & $6.62 \pm 1.92$ & $120.06 \pm 28.54$ \\
\hline
\end{tabular}

All treated groups showed statistically no significant differences from the control group $(P>0.05)$.

ALP: alkaline phosphatase; ALT: alanine aminotransferase; AST: aspartate aminotransferase; BUN: blood urea nitrogen; UA: uric acid.

TABLE 3: Pharmacokinetic parameters of different doses of LDH nanoparticles in mice after oral administration.

\begin{tabular}{lcccrr}
\hline Dose & $C_{\max }(\mathrm{mg} / \mathrm{L})$ & $T_{\max }(\mathrm{h})$ & AUC $(\mathrm{h} \times \mathrm{mg} / \mathrm{L})$ & $T_{1 / 2}(\mathrm{~h})$ & $0.22 \pm 0.20$ \\
$5 \mathrm{mg} / \mathrm{kg}$ & $4.15 \pm 1.41$ & $0.08 \pm 0.00$ & $0.79 \pm 0.29$ & $0.73 \pm 0.49$ & $1.35 \pm 0.29$ \\
$50 \mathrm{mg} / \mathrm{kg}$ & $4.44 \pm 0.32$ & $0.14 \pm 0.10$ & $4.00 \pm 2.36$ & $8.74 \pm 4.05$ & $1.38 \pm 0.67$ \\
$300 \mathrm{mg} / \mathrm{kg}$ & $7.24 \pm 0.70$ & $0.17 \pm 0.10$ & $11.96 \pm 6.29$ & $1.86 \pm 0.10$ & $2.15 \pm 1.27$ \\
$2000 \mathrm{mg} / \mathrm{kg}$ & $9.36 \pm 1.92$ & $0.21 \pm 0.08$ & $3.14 \pm 0.57$ \\
\hline
\end{tabular}

$C_{\max }:$ maximum concentration; $T_{\max }$ : time to maximum concentration; AUC: area under the plasma concentration-time curve; $T_{1 / 2}$ : half-life; MRT: mean residence time.

nanoparticles could be absorbed into the blood stream via the gastrointestinal tract. About $15-30 \%$ of orally administered $\mathrm{ZnO}$ nanoparticles (2000 mg/kg), depending on surface charge, were determined to enter the systemic circulation [21], supporting low oral absorption of $\mathrm{LDH}$ nanoparticles compared to that of $\mathrm{ZnO}$ nanoparticles. It is worth noting that all MRT values were within $3.1 \mathrm{~h}$ even when $2000 \mathrm{mg} / \mathrm{kg}$ were administered, clearly indicating that the highest dose of LDH nanoparticles could be cleared from the body after $3.1 \mathrm{~h}$. The low absorption and rapid clearance kinetic profile of LDH nanoparticles may explain their low toxicity as observed in Figure 2 and Tables 1 and 2, implying their low chronic toxicity potential in after long-term exposure.

3.5. Tissue Distribution. Figure 4 shows the tissue distribution profile of $\mathrm{LDH}$ nanoparticles following single-dose oral administration in mice. LDH levels did not significantly increase in the kidney, liver, lung, and spleen for $60 \mathrm{~min}$ after oral administration compared to basal Mg level in the tissues. Statistically increased $\mathrm{Mg}$ concentrations were not found in the other organs, such as brain and heart (data not shown). Statistical analysis also confirmed that significant difference was not found between the control and LDH-treated organs after 1 and 14 days (data not shown). These results suggest that orally administered LDH nanoparticles did not significantly accumulate in any specific target tissue, which can be explained by their pharmacokinetic behaviors (Figure 3), such as rapid decrease in the plasma concentration-time curve, low absorption, and short residence time. On the other hand, tissue distribution of inorganic nanoparticles to target organs has been generally reported after oral administration; the kidney, liver, and lung were found to be target organs for accumulation of $\mathrm{ZnO}$ nanoparticles, regardless of particle size, surface charge, or gender [22], while silver nanoparticles were primarily distributed to the liver following single-dose oral administration to rats [23]. Hence, the fact that $\mathrm{LDH}$ nanoparticles were not found in any specific target tissue strongly supports their low toxicity potential.

3.6. Excretion Profile. Excretion patterns of LDH nanoparticles via urine and feces following oral administration are presented in Figure 5. Significantly increased $\mathrm{Mg}$ levels were found in urine and feces within $24 \mathrm{~h}$ but returned to normal levels after $24 \mathrm{~h}$. A much higher $\mathrm{Mg}$ concentration was detected in feces than in urine, indicating that most $\mathrm{LDH}$ nanoparticles were eliminated via feces. This result also suggests that direct fecal clearance and biliary excretion play a major role in the elimination of $\mathrm{LDH}$ nanoparticles. 


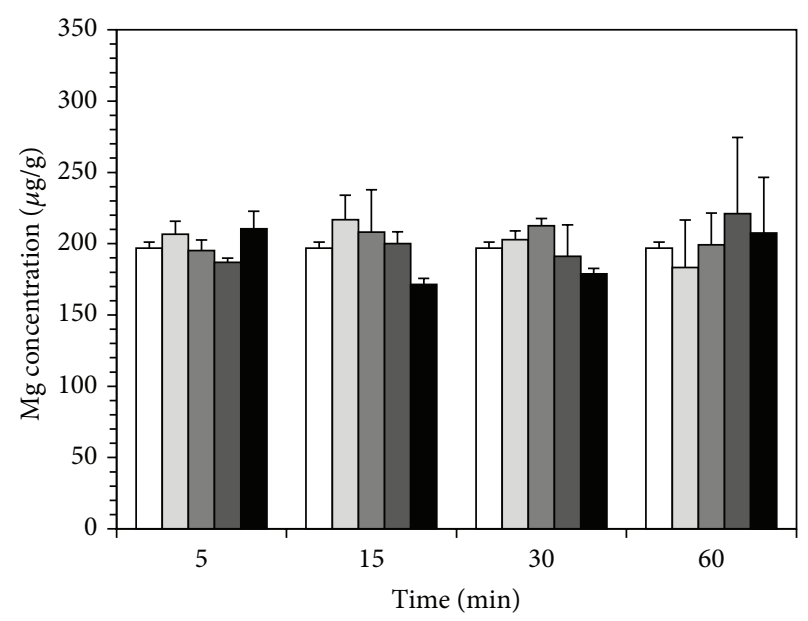

(a)

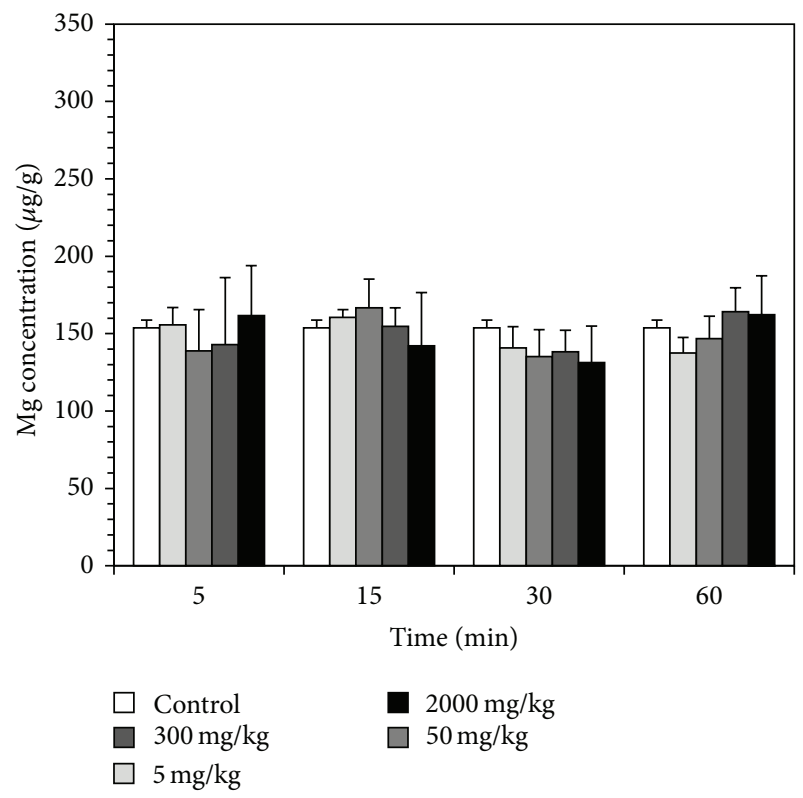

(c)

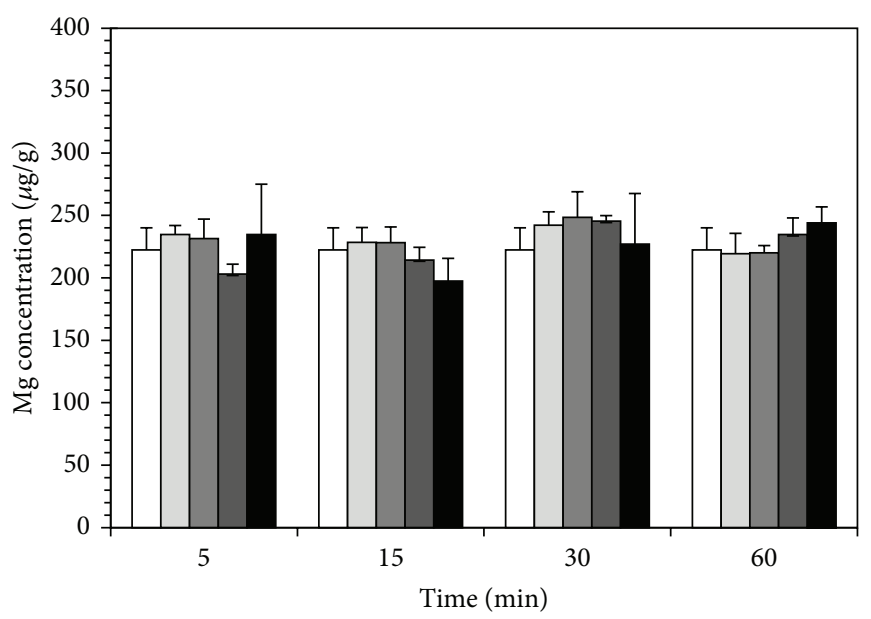

(b)

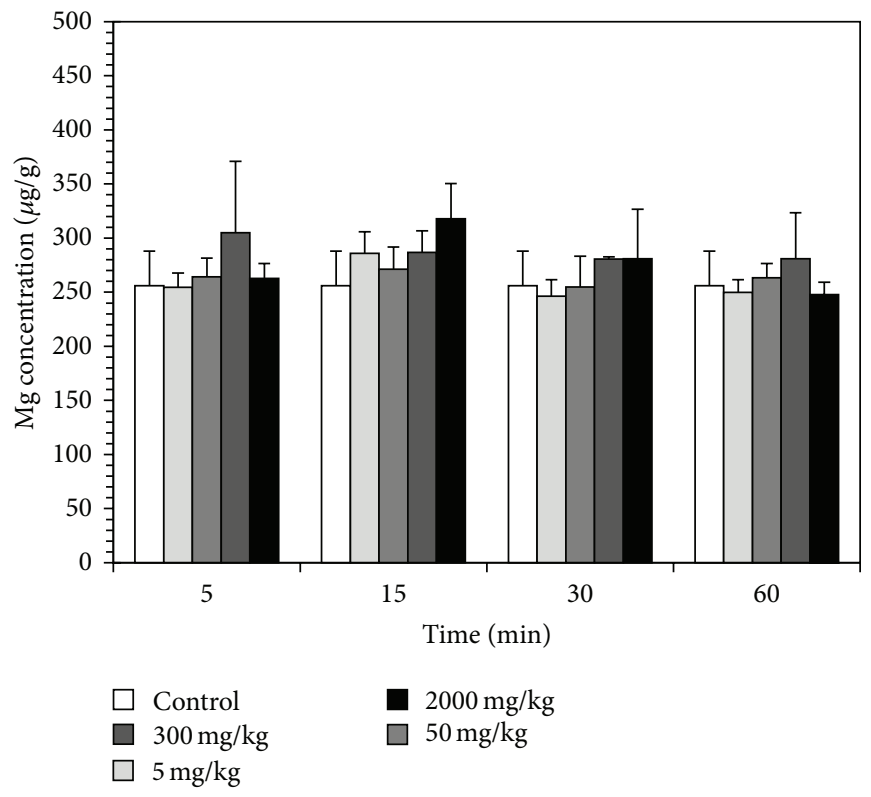

(d)

FIGURE 4: Tissue distribution of LDH nanoparticles after single-dose oral administration to mice. (a) Kidney, (b) liver, (c) lung, and (d) spleen. No significant significance was found between the control and LDH-treated groups.

\section{Conclusion}

The acute oral toxicity of LDH nanoparticles of about $100 \mathrm{~nm}$ was evaluated after administration of four different doses (5, 50,300 , and $2000 \mathrm{mg} / \mathrm{kg}$ ) in mice. The results demonstrated that LDH nanoparticles did not cause any mortality, abnormal behaviors, symptoms, and body weight loss up to the highest dose administered, indicating that the $\mathrm{LD}_{50}$ values were more than $2000 \mathrm{mg} / \mathrm{kg}$. Organosomatic indices of all organs in mice treated with $\mathrm{LDH}$ nanoparticles were not affected, thereby clearly supporting their low oral toxicity. Serum biochemical parameters did not significantly increase, suggesting that $\mathrm{LDH}$ nanoparticles did not induce liver or kidney injury. Pharmacokinetic and tissue distribution studies showed that $\mathrm{LDH}$ nanoparticles up to the dose of
$2000 \mathrm{mg} / \mathrm{kg}$ were rapidly absorbed within $30 \mathrm{~min}$ and cleared from the body after $3.1 \mathrm{~h}$, without significant accumulation in any specific target tissue. LDH nanoparticles were determined to be excreted via urine and feces within $24 \mathrm{~h}$. Taken together, it is concluded that LDH nanoparticles do not cause acute oral toxicity in mice, which is strongly associated with their small absorbed amount by the gastrointestinal system and easy clearance kinetics at the systemic level.

\section{Conflict of Interests}

The authors declare that there is no conflict of interests regarding the publication of this paper. 


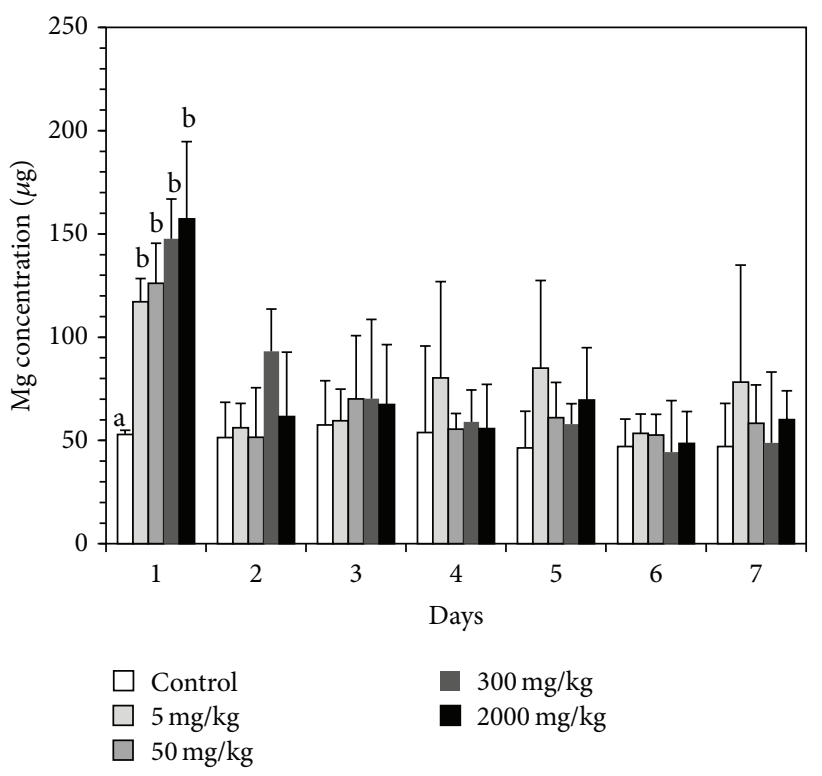

(a)

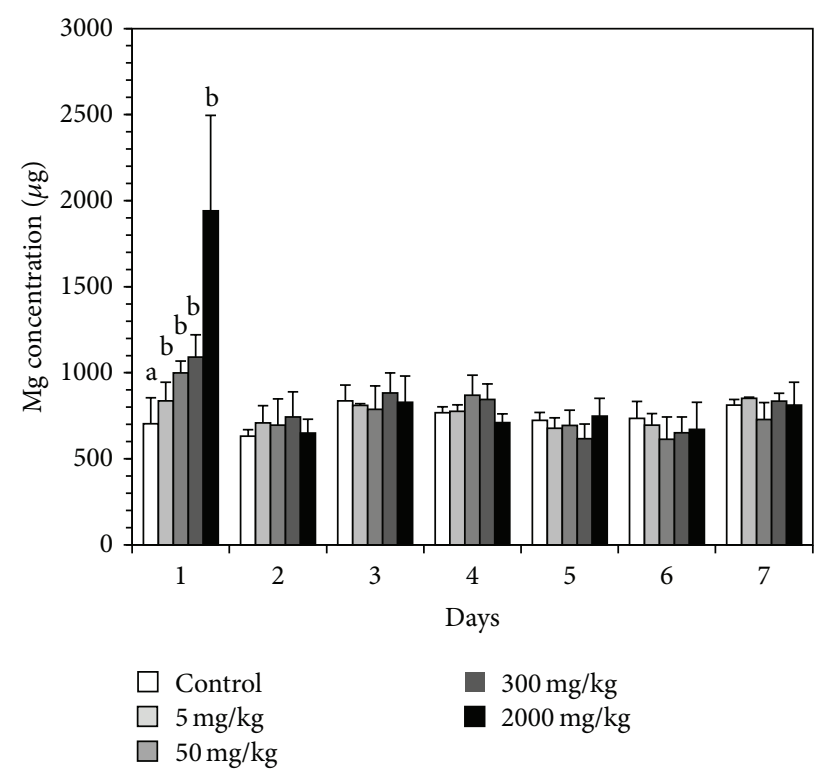

(b)

FIGURE 5: Excretion profiles of LDH nanoparticles via urine (a) and feces (b) after single-dose oral administration to mice.

\section{Acknowledgments}

This research was supported by the Basic Science Research Program through the National Research Foundation of Korea (NRF) funded by the Ministry of Education, Science and Technology (2010-0003570) and the National Research Foundation of Korea (NRF) Grant funded by the Korean government (MSIP) (2005-0049412) and partly by the National Research Foundation of Korea Grant funded by the Korean Government (KRF-2008-313-F00130).

\section{References}

[1] D. P. Cormode, T. Skajaa, Z. A. Fayad, and W. J. M. Mulder, "Nanotechnology in medical imaging: probe design and applications," Arteriosclerosis, Thrombosis, and Vascular Biology, vol. 29, no. 7, pp. 992-1000, 2009.

[2] X. Li, L. Wang, Y. Fan, Q. Feng, F. Z. Cui, and F. Watari, "Nanostructured scaffolds for bone tissue engineering," Journal of Biomedical Materials Research A, vol. 101, no. 8, pp. 24242435, 2013.

[3] A. C. Bonoiu, S. D. Mahajan, H. Ding et al., "Nanotechnology approach for drug addiction therapy: gene silencing using delivery of gold nanorod-siRNA nanoplex in dopaminergic neurons," Proceedings of the National Academy of Sciences of the United States of America, vol. 106, no. 14, pp. 5546-5550, 2009.

[4] J. Fan, G. Fang, X. Wang, F. Zeng, Y. Xiang, and S. Wu, “Targeted anticancer prodrug with mesoporous silica nanoparticles as vehicles," Nanotechnology, vol. 22, no. 45, Article ID 455102, 2011.

[5] A. Vaccari, "Preparation and catalytic properties of cationic and anionic clays," Catalysis Today, vol. 41, no. 1-3, pp. 53-71, 1998.

[6] H. Morioka, H. Tagaya, M. Karasu, J.-I. Kadokawa, and K. Chiba, "Preparation of hydroxy double salts exchanged by organic compounds," Journal of Materials Research, vol. 13, no. 4, pp. 848-851, 1998.

[7] Z. P. Xu, Q. H. Zeng, G. Q. Lu, and A. B. Yu, "Inorganic nanoparticles as carriers for efficient cellular delivery," Chemical Engineering Science, vol. 61, no. 3, pp. 1027-1040, 2006.

[8] J.-H. Choy, S.-J. Choi, J.-M. Oh, and T. Park, "Clay minerals and layered double hydroxides for novel biological applications," Applied Clay Science, vol. 36, no. 1-3, pp. 122-132, 2007.

[9] J.-M. Oh, S.-J. Choi, S.-T. Kim, and J.-H. Choy, "Cellular uptake mechanism of an inorganic nanovehicle and its drug conjugates: enhanced efficacy due to clathrin-mediated endocytosis," Bioconjugate Chemistry, vol. 17, no. 6, pp. 1411-1417, 2006.

[10] J.-M. Oh, S.-J. Choi, G.-E. Lee, J.-E. Kim, and J.-H. Choy, "Inorganic metal hydroxide nanoparticles for targeted cellular uptake through clathrin-mediated endocytosis," Chemistry, vol. 4, no. 1, pp. 67-73, 2009.

[11] S.-J. Choi and J.-H. Choy, "Layered double hydroxide nanoparticles as target-specific delivery carriers: uptake mechanism and toxicity," Nanomedicine, vol. 6, no. 5, pp. 803-814, 2011.

[12] S.-J. Choi, J.-M. Oh, and J.-H. Choy, "Toxicological effects of inorganic nanoparticles on human lung cancer A549 cells," Journal of Inorganic Biochemistry, vol. 103, no. 3, pp. 463-471, 2009.

[13] S.-J. Choi, J.-M. Oh, and J.-H. Choy, "Safety aspect of inorganic layered nanoparticles: Size-dependency in vitro and in vivo," Journal of Nanoscience and Nanotechnology, vol. 8, no. 10, pp. 5297-5301, 2008.

[14] S.-J. Choi, J.-M. Oh, T. Park, and J.-H. Choy, "Cellular toxicity of inorganic hydroxide nanoparticles," Journal of Nanoscience and Nanotechnology, vol. 7, no. 11, pp. 4017-4020, 2007.

[15] M. Baek, I.-S. Kim, J. Yu, H. E. Chung, J.-H. Choy, and S.-J. Choi, "Effect of different forms of anionic nanoclays on cytotoxicity," Journal of Nanoscience and Nanotechnology, vol. 11, no. 2, pp. 1803-1806, 2011. 
[16] S.-J. Choi and J.-H. Choy, "Effect of physico-chemical parameters on the toxicity of inorganic nanoparticles," Journal of Materials Chemistry, vol. 21, no. 15, pp. 5547-5554, 2011.

[17] H. Meng, Z. Chen, G. Xing et al., "Ultrahigh reactivity provokes nanotoxicity: explanation of oral toxicity of nano-copper particles," Toxicology Letters, vol. 175, no. 1-3, pp. 102-110, 2007.

[18] J. Wang, G. Zhou, C. Chen et al., "Acute toxicity and biodistribution of different sized titanium dioxide particles in mice after oral administration," Toxicology Letters, vol. 168, no. 2, pp. 176185, 2007.

[19] K. M. Kim, Y. J. Lee, Y. G. Hong, and J. S. Kang, "Oral acute and subacute toxicity studies of decursin and decursinol angelate of Angelica gigas Nakai," Molecular and Cellular Toxicology, vol. 5, no. 2, pp. 153-159, 2009.

[20] J. Chen, X. Dong, J. Zhao, and G. Tang, "In vivo acute toxicity of titanium dioxide nanoparticles to mice after intraperitioneal injection," Journal of Applied Toxicology, vol. 29, no. 4, pp. 330337, 2009.

[21] M. Baek, H. E. Chung, J. Yu et al., "Pharmacokinetics, tissue distribution, and excretion of zinc oxide nanoparticles," International Journal of Nanomedicine, vol. 7, pp. 3081-3097, 2012.

[22] H. J. Paek, Y. J. Lee, H. E. Chung et al., "Modulationof the pharmacokinetics of zinc oxide nanoparticles and their fates in vivo," Nanoscale. 2013.

[23] K. Park, E.-J. Park, I. K. Chun et al., "Bioavailability and Toxicokinetics of citrate-coated silver nanoparticles in rats," Archives of Pharmacal Research, vol. 34, no. 1, pp. 153-158, 2011. 

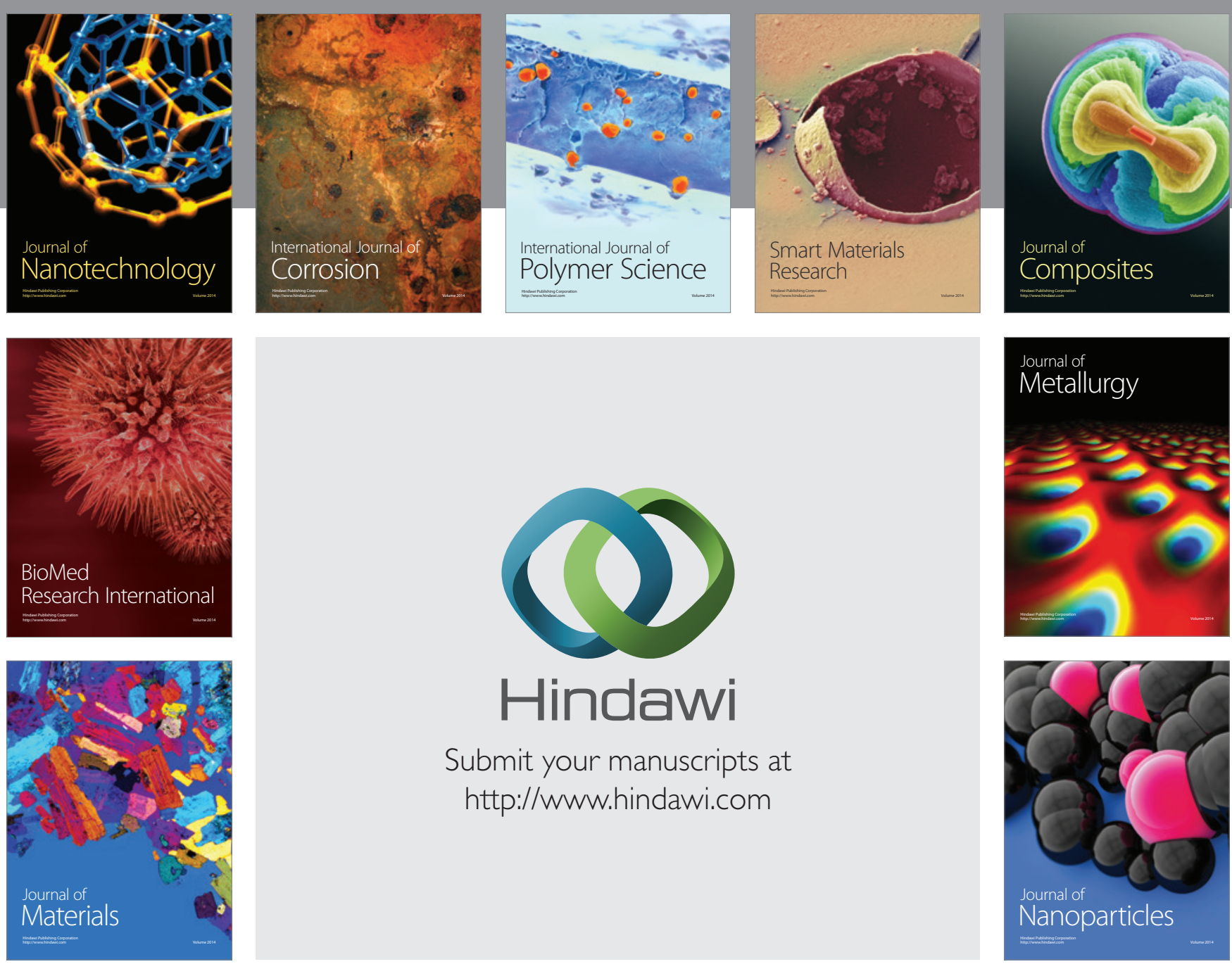

Submit your manuscripts at http://www.hindawi.com
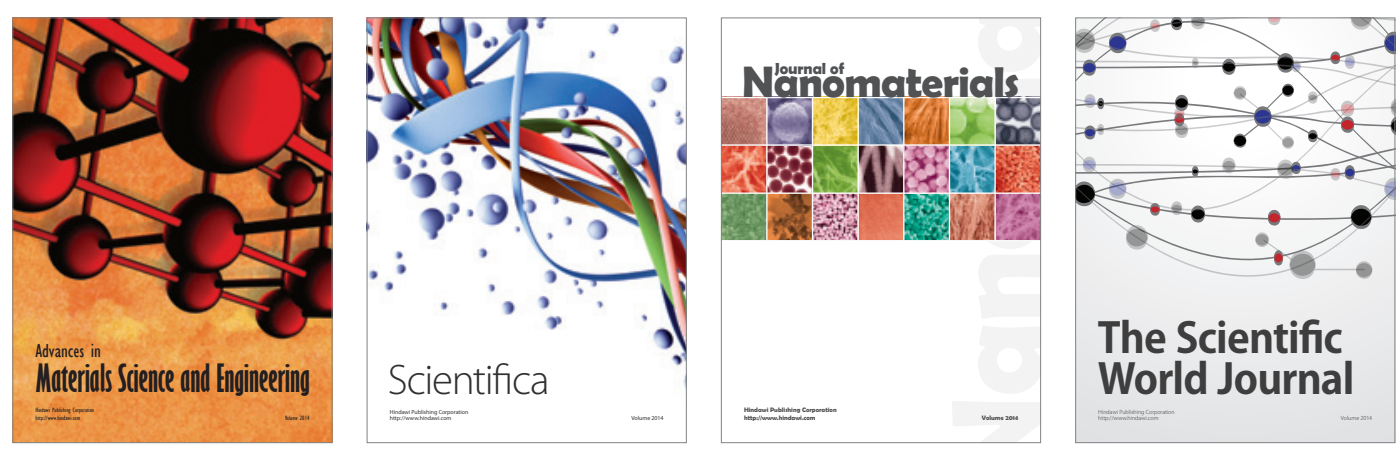

\section{The Scientific World Journal}
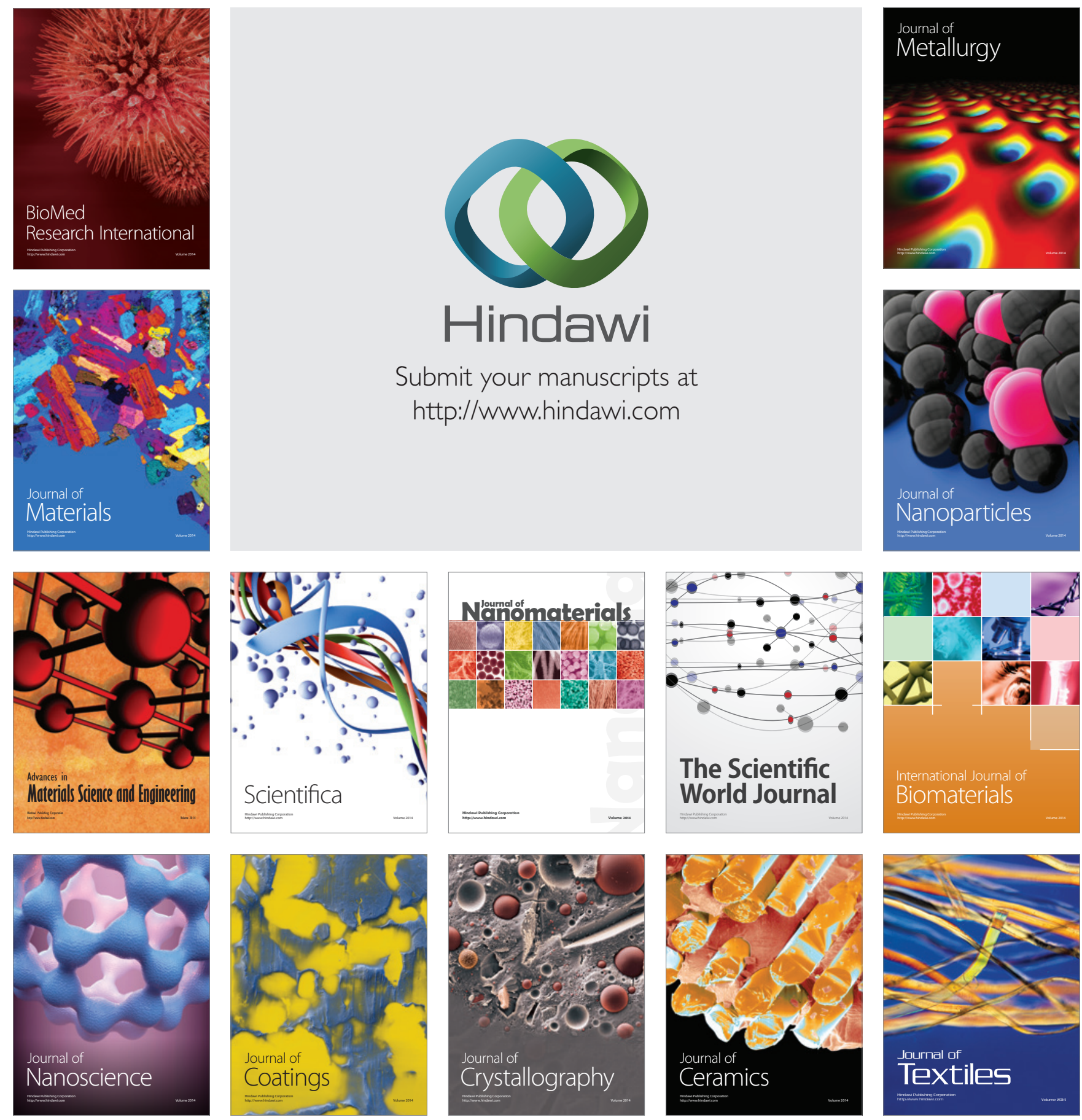